\title{
Hybrid Higgs inflation: The use of disformal transformation
}

\author{
Seiga Sato* and Kei-ichi Maeda ${ }^{\dagger}$ \\ Department of Physics, Waseda University, Shinjuku, Tokyo 169-8555, Japan
}

(Received 20 December 2017; published 17 April 2018)

\begin{abstract}
We propose a hybrid type of the conventional Higgs inflation and new Higgs inflation models. We perform a disformal transformation into the Einstein frame and analyze the background dynamics and the cosmological perturbations in the truncated model, in which we ignore the higher-derivative terms of the Higgs field. From the observed power spectrum of the density perturbations, we obtain the constraint on the nonminimal coupling constant $\xi$ and the mass parameter $M$ in the derivative coupling. Although the primordial tilt $n_{s}$ in the hybrid model barely changes, the tensor-to-scalar ratio $r$ moves from the value in the new Higgs inflationary model to that in the conventional Higgs inflationary model as $|\xi|$ increases. We confirm our results by numerical analysis by ADM formalism of the full theory in the Jordan frame.
\end{abstract}

DOI: $10.1103 /$ PhysRevD.97.083512

\section{INTRODUCTION}

Inflation was first proposed to solve the flatness and the horizon problem as well as the monopole problem in the early Universe [1-3]. It also predicts the origin of cosmological perturbations [4-7], which turns out to be the most important outcome for the observational confirmation of the idea. Since the generic predictions of the inflationary scenario have matched observations, inflation has now become a standard model of the early Universe.

Many inflation models have been proposed so far [8], but the constraints from the recent $\mathrm{CMB}$ observations are very severe. The density perturbations are almost scale invariant, while the tensor-to-scalar ratio should be very small. As a result, some inflation models already have been excluded.

In most inflationary scenarios, we assume the existence of a scalar field, called an inflaton, that is responsible for inflation. However we still do not know what the inflaton is. Since we know only one scalar field in the standard model, which is the Higgs particle, many models have looked for an inflaton beyond the standard model of particle physics because the Higgs field cannot be an inflaton to explain the observed density perturbations.

However, there is one loophole, which is the introduction of gravitational couplings to the Higgs field. When we quantize matter fields in a curved spacetime, we may find nonminimal coupling of the fields with a curvature. Including such a coupling term $\xi \phi^{2} R$, where $\xi$ is a nonminimal coupling constant and $R$ is a scalar curvature, some inflationary scenarios have been discussed [9-11]. Assuming this scalar field is the Higgs particle, which is only one scalar field in the standard model, the so-called

\footnotetext{
*s.seiga@gravity.phys.waseda.ac.jp

†maeda@waseda.jp
}

Higgs inflation has been proposed [12-16]. We call it the conventional Higgs inflation model. If $\xi$ is large negative, e.g., $\xi \sim-10^{4}$, this model provides a consistent inflationary scenario with observations.

New Higgs inflation was also proposed in 2010, which has a derivative coupling of the Higgs field with the Einstein curvature as $\frac{1}{2 M^{2}} G^{\mu \nu} \nabla_{\mu} \phi \nabla_{\nu} \phi$ [17,18]. When $M \sim 10^{-7} M_{\mathrm{P}}$, where $M_{\mathrm{P}}$ is the reduced Planck mass, this model is still consistent with observations. Although this model produces a rather large amount of the gravitational waves, the predicted tensor-to-scalar ratio is still just on the border of the observational constraints.

The common feature of both Higgs inflation models is no introduction of any additional degrees of freedom. Although we still do not know the physics at the very high-energy scale around the Planck scale $\left(\sim 10^{18} \mathrm{GeV}\right)$, the above coupling terms may appear at the high-energy scale [19]. Hence, it may be natural to consider a hybrid type of the above two inflation models.

Since the typical energy scale of inflation is very high, we have to take into account the quantum loop effects, which may destabilize the Higgs field [20-30]. Whether it is really unstable or stable may depend highly on the top quark mass. Even if it is stable, since the running coupling constants via renormalization depend on the energy scale, the effective potential will be modified much [14,15,31-36].

Moreover, the loss of unitarity in the tree-level scattering at the high-energy scale is also a serious problem. It is believed that it means the theory is nonrenormalizable and some new physics is required at such a scale. In general relativity, the scale is around the Planck scale. However, nonrenormalizable terms such as the nonminimal gravitational coupling terms in the conventional and new Higgs inflation would lower this scale. See for example the conventional Higgs inflation [37-43] and new Higgs inflation [44-46]. 
Although we may have to analyze our model with the loop corrections and find the solution of the unitarity problem, since the discussion on the quantum corrections is still in a fog, we will analyze the original tree model in this paper to show how the tensor-to-scalar ratio depends on the coupling parameters.

This paper is organized as follows. After introduction of the hybrid type Higgs inflation, we present our truncated model based on a disformal transformation into the Einstein frame and analyze the background cosmological dynamics in Sec. II. We confirm the validity of our truncation by the results calculated in the original full theory. In Sec. III, we analyze the cosmological perturbations and give the primordial tilt $n_{s}$ and the tensor-to-scalar ratio $r$. We then show the accuracies of our results obtained in the truncated model, which confirm our approach. Summary and discussion follow in Sec. IV. In Appendix A, we present the explicit forms of the disformal transformation and the higher-derivative terms of the Higgs field. We then give the basic equations for the background universe in the original Jordan frame in Appendix B. We also describe how to calculate the cosmological perturbations in the full theory by use of ADM formalism.

In this paper we use the reduced Planck units of $M_{\mathrm{P}}:=$ $(8 \pi G)^{-1 / 2}=1$ as well as $\hbar=c=1$.

\section{HYBRID HIGGS INFLATION}

First we summarize the original two Higgs inflation models and then extend them to a hybrid type.

\section{A. Two original Higgs inflation models}

The action of the conventional Higgs inflation based on nonminimal coupling of the Higgs field with a scalar curvature [12] is given by

$$
S_{J}=\int d^{4} x \sqrt{-g}\left[\frac{1-\xi \phi^{2}}{2} R(g)-\frac{1}{2}(\nabla \phi)^{2}-V(\phi)\right],
$$

where the potential of the Higgs field $\phi$ is given by

$$
V(\phi)=\frac{\lambda}{4}\left(\phi^{2}-v^{2}\right)^{2} .
$$

$\lambda$ is the Higgs self-coupling and $v(\approx 246 \mathrm{GeV})$ is the Higgs vacuum expectation value (VEV) [12]. When we discuss inflation, which energy scale may be much higher than $100 \mathrm{GeV}$, we can approximate the potential as

$$
V(\phi)=\frac{\lambda}{4} \phi^{4} .
$$

For the successful Higgs inflation, we assume $\xi<0$. Note that the sign of the nonminimal coupling $\xi$ is different from that in [12].
Performing a conformal transformation

$$
g_{\mu \nu}=\left(1-\xi \phi^{2}\right)^{-1} \bar{g}_{\mu \nu},
$$

we find the Einstein frame action

$$
S_{E}=\int d^{4} x \sqrt{-\bar{g}}\left[\frac{1}{2} R(\bar{g})-\frac{1}{2}(\bar{\nabla} \Phi)^{2}-U(\Phi)\right],
$$

where the variables with a bar denote those in the Einstein frame. $\Phi$ is the canonically normalized Higgs field, defined by

$$
\frac{d \Phi}{d \phi}=\frac{\sqrt{1-\xi(1-6 \xi) \phi^{2}}}{\left(1-\xi \phi^{2}\right)},
$$

and

$$
U(\Phi)=\frac{\lambda \phi^{4}}{4\left(1-\xi \phi^{2}\right)^{2}}
$$

is the potential in the Einstein frame.

When $|\xi| \gg 1$, we find

$$
\Phi \approx \sqrt{\frac{3}{2}} \ln \left(1-\xi \phi^{2}\right),
$$

which gives

$$
U(\Phi) \approx \frac{\lambda}{4 \xi^{2}}\left(1-e^{-\sqrt{\frac{2}{3}} \Phi}\right)^{2} .
$$

This model is almost equivalent to the Starobinsky inflation with the curvature squared term [1]. When we perform the conformal transformation [47,48], the model is described by the Einstein gravity plus a scalar field with the same potential.

This potential approaches a constant exponentially as $\Phi \rightarrow \infty$, which guarantees de Sitter expansion. Assuming $\lambda \simeq 0.1$, if $\xi \sim-10^{4}$, we obtain the cosmological perturbations consistent with observations as the primordial tilt $n_{s} \sim 0.96$ and the tensor-to-scalar ratio $r \sim 10^{-3}$ as well as the power spectrum of the density perturbations $\mathcal{P}_{\zeta} \sim 10^{-9}$ [12].

An alternative approach, called new Higgs inflation, adds a coupling between derivatives of the Higgs field with the Einstein curvature, which action is given by

$$
\begin{aligned}
S_{J}= & \int d^{4} x \sqrt{-g}\left[\frac{1}{2} R(g)\right. \\
& \left.-\frac{1}{2}\left(g^{\mu \nu}-\frac{G^{\mu \nu}}{M^{2}}\right) \nabla_{\mu} \phi \nabla_{\nu} \phi-V(\phi)\right] .
\end{aligned}
$$

where $G^{\mu \nu}$ is the Einstein tensor and $M$ is a coupling constant described by a characteristic mass scale [16-18]. 
Like the conventional Higgs inflation, this does not introduce extra degrees of freedom. The new coupling constant takes on a value $M \simeq 5 \times 10^{-8} \lambda^{-1 / 4}$ when normalized with $\mathrm{CMB}$ data. We find $n_{s} \sim 0.97$ and $r \sim 0.1$ [44].

\section{B. Hybrid Model}

Here we propose a hybrid type of the previous two Higgs inflation models [49]. The action we assume is

$$
\begin{aligned}
S_{J}= & \int d^{4} x \sqrt{-g}\left[\frac{1-\xi \phi^{2}}{2} R(g)\right. \\
& \left.-\frac{1}{2}\left(g^{\mu \nu}-\frac{G^{\mu \nu}}{M^{2}}\right) \nabla_{\mu} \phi \nabla_{\nu} \phi-V(\phi)\right],
\end{aligned}
$$

which we call a hybrid Higgs inflation model.

This type of model is classified into more general model proposed in [19], which they called generalized Higgs inflation. They discussed the model in the original Jordan frame. Since the basic equations are complicated, some limiting case was discussed to present a simple formula for the cosmological perturbations. However it turns out that their approximation cannot be applied to the conventional Higgs inflation model (see later).

Hence in this paper we shall perform a disformal transformation to the Einstein frame and ignore the higher-derivative terms[50], which may be justified during the slow-rolling inflationary period as we will show the detail later. We show how to get the Einstein frame via a disformal transformation in Appendix A.

The action (2.1) in the Einstein frame is described as

$$
\begin{aligned}
S_{E}= & \int d^{4} x \sqrt{-\bar{g}}\left[\frac{1}{2} \bar{R}-\frac{1}{2}(\bar{\nabla} \Phi)^{2}-U(\Phi)\right. \\
& + \text { higher derivative terms }]
\end{aligned}
$$

where the variables with a bar denote those in the Einstein frame. New canonically normalized scalar field $\Phi$ is defined by

$$
\frac{d \Phi}{d \phi}=\left(1-\xi \phi^{2}\right)^{-1} \sqrt{1-\xi(1-6 \xi) \phi^{2}+\frac{V}{M^{2}}} .
$$

The effective potential in the Einstein frame $U(\Phi)$ is given by

$$
U(\Phi)=\frac{V(\phi)}{\left(1-\xi \phi^{2}\right)^{2}},
$$

in the form of the parametric representation by the original Higgs field $\phi$. Fig. 1 shows the schematic shape of $U(\Phi)$.

In the transformed action (2.2), there are many higher derivative terms such as $(\nabla \phi)^{4}$ and $\square \phi$. In Appendix A, we present the higher-derivative terms explicitly. However,

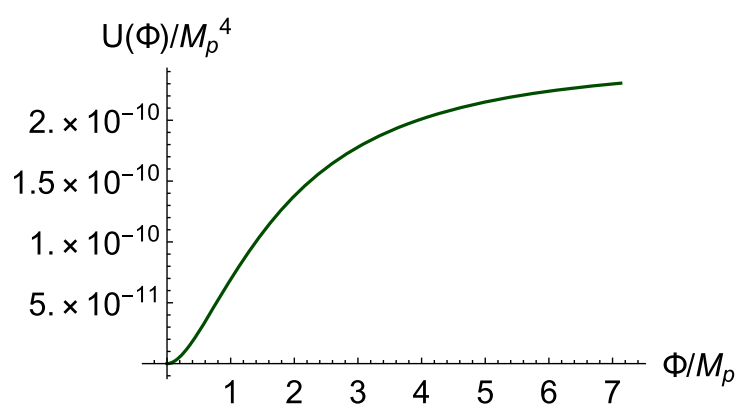

FIG. 1. The effective potential $U(\Phi)$ in the Einstein frame for the coupling parameters $M=10^{-7}, \xi=-10^{4}$ and $\lambda=0.1$.

since we discuss the inflationary period, in which the inflaton changes slowly, we may be able to ignore those terms. The resultant truncated action is now

$$
S_{E}^{(\mathrm{T})}=\int d^{4} x \sqrt{-\bar{g}}\left[\frac{1}{2} \bar{R}-\frac{1}{2}(\bar{\nabla} \Phi)^{2}-U(\Phi)\right] .
$$

If this action approximates the original one well, we can easily analyze the inflationary stage just by the potential $U(\Phi)$. This is the great advantage of the use of a disformal transformation. This potential is expanded in the large field limit as

$$
U(\Phi)=\frac{\lambda}{4 \xi^{2}}\left[1-\frac{\lambda}{2|\xi|^{3} M^{2}} \frac{1}{\Phi^{2}}+\cdots\right] .
$$

This inflation potential shape is favored by the CMB observation because it is concave. This potential asymptotically converges to be a flat plateau in the large field region as $\Phi^{-2}$ unlike the conventional Higgs inflation, which approaches a constant exponentially as $\exp \left(-2 \sqrt{\frac{2}{3}} \Phi\right)$.

In what follows, we analyze this truncated model and then justify this approach by comparing with the results obtained without truncation in the original Jordan frame.

\section{Background Dynamics}

In this subsection, we first analyze an isotropic and homogeneous universe model. We assume that the metric form is given by the flat FLRW (Friedmann-LemâitreRobertson-Walker) spacetime as

$$
d \bar{s}^{2}=-d \bar{t}^{2}+\bar{a}^{2} d \overline{\boldsymbol{x}}^{2} .
$$

When we ignore the higher-derivative terms, the basic equations in the Einstein frame are given by

$$
\begin{gathered}
\bar{H}^{2}=\frac{1}{3}\left[\frac{1}{2}\left(\frac{d \Phi}{d \bar{t}}\right)^{2}+U(\Phi)\right] \\
\dot{\bar{H}}+\bar{H}^{2}=-\frac{1}{3}\left[\left(\frac{d \Phi}{d \bar{t}}\right)^{2}-U(\Phi)\right]
\end{gathered}
$$




$$
\frac{d^{2} \Phi}{d \bar{t}^{2}}+3 \bar{H} \frac{d \Phi}{d \bar{t}}+\frac{d U}{d \Phi}=0
$$

where the Hubble expansion parameter is defined by

$$
\bar{H}=\frac{1}{\bar{a}} \frac{d \bar{a}}{d \bar{t}} .
$$

Since the potential $U(\Phi)$ in the Einstein frame has a very flat plateau at the large field range, we will have a slow-roll inflation. Once the scalar field starts to roll the potential very slowly, the higher-derivative terms can be ignored. As a result, we expect the truncated model (2.3) may approximate an inflationary period very well.

In order to justify this expectation, we solve both cosmological dynamics in the original model and in the truncated model, and compare those results. Since the full equations in the Einstein frame are too complicated, we solve them in the original Jordan frame, which basic equations are given in Appendix B 1. Equations (B1), (B2), and (B3) in the original Jordan frame correspond to Eqs (2.4), (2.5), and (2.6), respectively.

Note that the cosmic time $\bar{t}$ in the Einstein frame is different from the cosmic time $t$ in the Jordan frame. Since two metrics are related as

$$
\begin{aligned}
d s^{2} & =g_{\mu \nu} d x^{\mu} d x^{\nu}=-d t^{2}+a^{2} d x^{2} \\
& =\left(1-\xi \phi^{2}\right)^{-1}\left[d \bar{s}^{2}+\frac{\nabla_{\mu} \phi \nabla_{\nu} \phi}{2 M^{2}} d x^{\mu} d x^{\nu}\right] \\
& =\left(1-\xi \phi^{2}\right)^{-1}\left[-d \bar{t}^{2}+\bar{a}^{2} d \overline{\boldsymbol{x}}^{2}+\frac{\dot{\phi}^{2}}{2 M^{2}} d t^{2}\right],
\end{aligned}
$$

where a dot denotes the derivative with respect to the cosmic time $t$ in the Jordan frame, we find the relations between two cosmic times ( $t$ and $\bar{t}$ ) and two scale factors $(a$ and $\bar{a})$ as

$$
\begin{aligned}
d \bar{t}^{2} & =\left(1-\xi \phi^{2}+\frac{\dot{\phi}^{2}}{2 M^{2}}\right) d t^{2} \\
\bar{a}^{2} & =\left(1-\xi \phi^{2}\right) a^{2}
\end{aligned}
$$

respectively.

We numerically solve the basic equations, and compare both dynamics. Fig. 2 shows the time evolution of the Hubble expansion parameter $H$ in the Jordan frame. In stead of the cosmic time to describe the evolution, we use the $e$-folding number $N$ to the end of inflation, which is defined by

$$
N=\ln \left(\frac{a_{\text {end }}}{a}\right),
$$

where $a_{\text {end }}$ is the scale factor at the end of inflation. In order to compare the numerical solutions, the solution of the truncated model in the Einstein frame is transformed to the Jordan-frame variables.

Although there exists some small deviation at the end of inflation $(N=0)$, when the scalar field starts to move

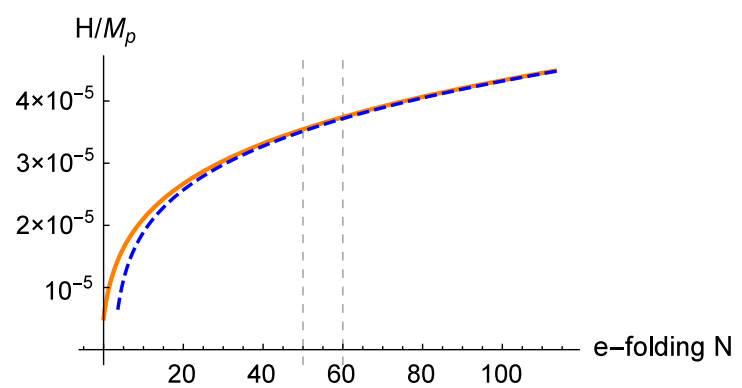

FIG. 2. The background dynamics for the coupling parameters $M=10^{-7}, \xi=-10^{4}$. We show the evolution of the Hubble expansion parameter $H$ with respect to the $e$-folding number $N$ in the Jordan frame. The orange curve denotes the evolution of the full theory in the Jordan frame, while the blue dotted curve shows the results obtained by the truncated model in the Einstein frame. We transform its solution into the Jordan frame variables. We also show the reference $e$-folding number of $N=50-60$ by the dotted light-gray lines.

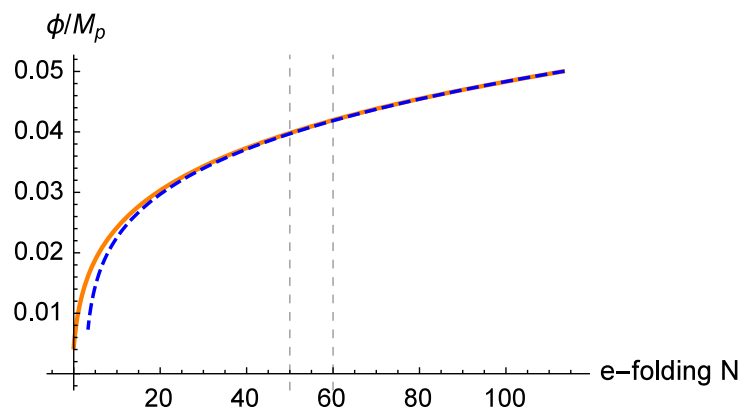

FIG. 3. The evolution of the Higgs field $\phi$ with respect to the $e$-folding number $N$. The coupling parameters, the colored curves, and the dotted light-gray lines are the same setting as those in Fig. 2.



FIG. 4. The phase-space evolution of the Higgs field $(\phi-\dot{\phi})$. The coupling parameters, the colored curves, and the dotted lightgray lines are the same setting as those in Fig. 2.

rapidly, the truncated model approximates the original one very well until $N \approx 20$. Especially around $N=50-60$, when the observed density perturbations are produced, the agreement is quite good. 
We also depict the evolution of the Higgs field $\phi$ and its phase diagram (the $\phi-\dot{\phi}$ diagram) in Figs. 3 and 4 , respectively. These also support the validity of our truncation. In the early stage of inflation, both background solutions are almost the same. At the end of inflation, the deviation appears since the higher-derivative terms seem no longer negligible.

In the next section, we will discuss the cosmological perturbations and the validity of the truncated model.

\section{COSMOLOGICAL PERTURBATIONS}

\section{A. Perturbations in the truncated model}

In order to confirm our inflationary model, we have to evaluate the cosmological perturbations and compare them with the observed data. As shown in [51-56], a disformal transformation as well as a conformal transformation will not change the cosmological perturbations. There exists the invariance of the cosmological perturbations under such transformations. Hence, we can evaluate the perturbations either in the Einstein frame or in the Jordan frame. Since the full equations in the Einstein frame are very complicated, we usually analyze the dynamics in the original Jordan frame. In fact, in generalized Higgs inflation model, which includes ours, they have analyzed the perturbations in the Jordan frame [19]. Since the full analysis of perturbations is tedious, they have also provided one simple formula under some approximation [19].

However, as shown in the subsection II C, during the inflationary stage when the scalar field changes very slowly, the background dynamics in the truncated model well approximates that in the original full model. Hence we expect that the cosmological perturbations in the truncated model may also provide a good approximation to the original ones.

Here we first evaluate the cosmological perturbations in the truncated model in the Einstein frame, in which the perturbations can be obtained easily just by the effective potential $U(\Phi)$. This is a great advantage of the present method.

The potential slow-roll parameters are defined by

$$
\begin{aligned}
& \epsilon=\frac{1}{2}\left(\frac{1}{U} \frac{d U}{d \Phi}\right)^{2}, \\
& \eta=\frac{1}{U} \frac{d^{2} U}{d \Phi^{2}} .
\end{aligned}
$$

The power spectrum of the density perturbations $\mathcal{P}_{\zeta}$, its spectral index $n_{s}$ and the tensor-scalar ratio $r$ are given by the slow-roll parameters as

$$
\begin{aligned}
\mathcal{P}_{\zeta} & \simeq \frac{1}{24 \pi^{2}} \frac{U}{\epsilon}, \\
n_{s} & \simeq 1-6 \epsilon+2 \eta, \\
r & \simeq 16 \epsilon .
\end{aligned}
$$

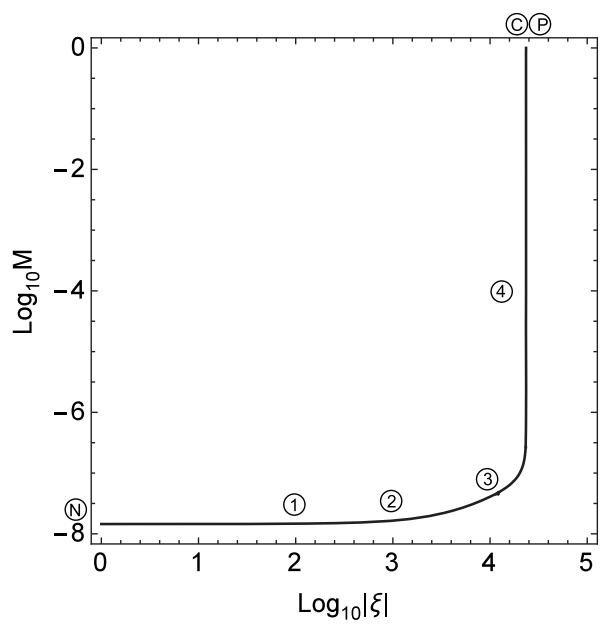

FIG. 5. The constraint on the coupling parameters $M$ and $\xi$. The color dots with numbers or letters correspond to the coupling parameters given in Table. I.

Applying them to the present model, the power spectrum of the density perturbations is written by

$$
\mathcal{P}_{\zeta} \simeq \frac{\lambda \phi^{6}\left(4 M^{2}+4 \xi M^{2}(6 \xi-1) \phi+\lambda \phi^{4}\right)}{3072 \pi^{2} M^{2}\left(1-\xi \phi^{2}\right)^{2}} .
$$

Since $U(\Phi)$ is not explicitly given by $\Phi$ but by some parametric representation of $\phi$, we have also used the original Higgs field $\phi$ as a "parameter" when we solve the dynamics.

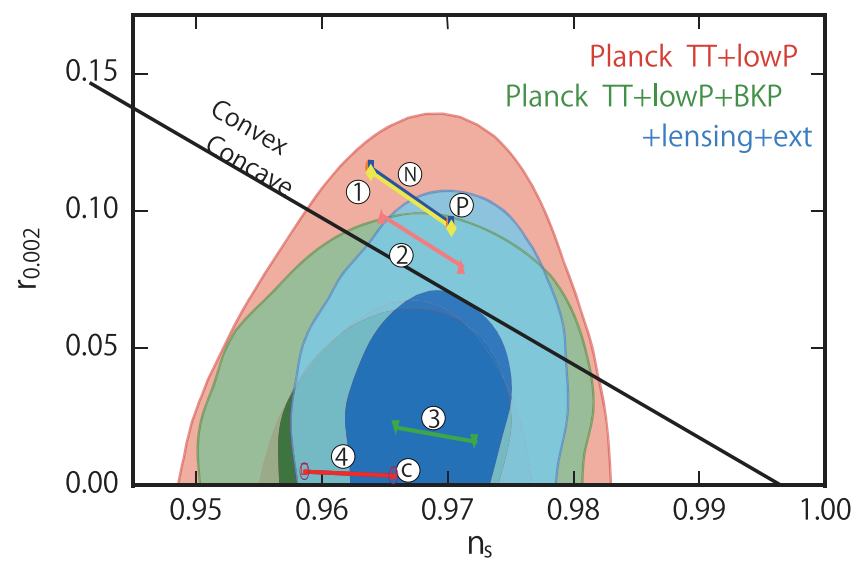

FIG. 6. The colored lines show the numerical results of the hybrid Higgs inflation with $N=50-60$. The numbers or letters of lines correspond to the colored dots in Fig. 5 and the coupling parameters in Table I. (N) and (C) correspond to new Higgs inflation model with $M=1.41 \times 10^{-8}$ and the conventional Higgs inflation with $\xi=-10^{4}$, respectively. ${ }^{P}$ shows the case with positive nonminimal coupling $(\xi=1 / 6)$, which line is overlapping with the line (N). (C) and (4) are almost the same. The observational constraints on $n_{s}$ and $r$ by Planck 2015 [57] are also shown. 
The $e$-folding number $N$ is given by

$$
N \simeq-\int_{\Phi_{\text {end }}}^{\Phi_{N}} \frac{U}{d U / d \Phi} d \Phi \simeq-\frac{\phi_{N}^{2} \xi\left\{8 M^{2} \xi^{2}(6 \xi-1)+\lambda\left(2+\phi_{N}^{2} \xi\right)\right\}+2\left(\lambda+24 M^{2} \xi^{3}\right) \ln \left[1-\xi \phi_{N}^{2}\right]}{64 M^{2} \xi^{3}},
$$

where $\Phi_{N}$ and $\phi_{N}$ are the values of $\Phi$ and $\phi$ at the $e$-folding number $N$, and $\Phi_{\text {end }}$ and $\phi_{\text {end }}$ are those values at the end of inflation $(\epsilon=1)$.

From the Planck CMB observation [57], we have the constraint on the power spectrum $\mathcal{P}_{\zeta} \sim 2.2 \times 10^{-9}$. Here we assume that $\Phi_{\text {end }}, \phi_{\text {end }}$ are much smaller than $\Phi_{N}, \phi_{N}$, and then we set $\Phi_{\text {end }}, \phi_{\text {end }}=0$. From Eq. (3.2), we obtain the value of the inflaton at $N=50-60$. Substituting these values into Eq. (3.1), we obtain the constraint on $\xi$ and $M$, which is shown by the black curve in Fig. 5 .

We then calculate $n_{s}$ and $r$, which results are plotted by the colored lines with numbers or letters in Fig. 6. We choose 7 sets of the coupling parameters:

(P) (orange), () (blue), (1) (yellow), (2) (pink),

(3) (green), (4) (purple), (C) (red),

which parameter values are given in Table I. The numbers or letters also correspond to the colored dots in Fig. 5. (, $\mathbb{1}$, and $\left({ }^{\circ}\right.$ give the models of the conventional Higgs inflation, of new Higgs inflation, and with a positive nonminimal coupling $(\xi=1 / 6)$, respectively.

We find that the predicted point $\left(n_{s}, r\right)$ in the hybrid Higgs inflation model moves from the values in new Higgs inflation model to those in the conventional Higgs inflationary model as $|\xi|$ increases. The tensor-to-scalar ratio $r$ changes drastically, while the primordial tilt $n_{s}$ barely changes. Hence once we know the tensor-scalar ratio $r$ by the future observations, we can fix the coupling parameters $M$ and $\xi$.

As found in Fig. 6, when we have the derivative coupling, even if there exists a positive nonminimal coupling constant $(\xi>0)$, e.g., a conformal coupling $(\xi=1 / 6)$, an inflationary scenario consistent with observations becomes possible[10,11]. The results are almost the same as new Higgs inflation model because nonminimal coupling does not play any important role except for the constraint on the initial value of the Higgs field $\left(\phi_{\text {in }}<\xi^{-1 / 2}\right)$. The large positive coupling may not give a successful inflation.

\section{B. Comparison with the "exact" results}

In order to justify our results calculated in the truncated model, we have to evaluate the perturbations in the original full theory. Since the basic equations in the Einstein frame is too complicated, we perform the calculation of perturbations in the original Jordan frame by use of the ADM formalism, which is summarized in Appendix B 2. We suppose that this numerical result gives the correct values. We then compare our results with those "exact" ones. In Table I, we show the "accuracies" of our results, which are evaluated by the following deviations;

TABLE I. The coupling parameters for which we evaluate the cosmological perturbations in the hybrid Higgs inflation model. We also show the accuracies of our results in our truncated model in the Einstein frame (THD) and those in the large friction limit (LFL) in Jordan frame. The accuracies in THD are always fairly good, while the accuracy in LFL becomes much worse as the coupling parameters approach the "conventional" Higgs inflation model, e.g. the model (4), because the approximation is no longer valid.

\begin{tabular}{|c|c|c|c|c|c|c|c|}
\hline & \multicolumn{2}{|c|}{ Coupling parameters } & \multirow{2}{*}{$\begin{array}{l}\text { Approximation } \\
\text { methods }\end{array}$} & \multicolumn{2}{|c|}{$\Delta n_{s} / n_{s}$} & \multicolumn{2}{|c|}{$\Delta r / r$} \\
\hline & $M$ & $\xi$ & & $N=60$ & $N=50$ & $N=60$ & $N=50$ \\
\hline$\overline{(P}$ & $1.41 \times 10^{-8}$ & $1 / 6$ & $\begin{array}{l}\text { THD } \\
\text { LFL }\end{array}$ & $\begin{array}{l}7.1 \times 10^{-4} \\
5.5 \times 10^{-4}\end{array}$ & $\begin{array}{l}1.0 \times 10^{-3} \\
7.5 \times 10^{-4}\end{array}$ & $\begin{array}{l}3.5 \times 10^{-2} \\
3.1 \times 10^{-2}\end{array}$ & $\begin{array}{l}4.5 \times 10^{-2} \\
3.1 \times 10^{-2}\end{array}$ \\
\hline (i) & $1.41 \times 10^{-8}$ & 0 & $\begin{array}{l}\text { THD } \\
\text { LFL }\end{array}$ & $\begin{array}{l}5.8 \times 10^{-2} \\
5.0 \times 10^{-2}\end{array}$ & $\begin{array}{l}9.3 \times 10^{-4} \\
7.6 \times 10^{-4}\end{array}$ & $\begin{array}{l}3.4 \times 10^{-2} \\
3.1 \times 10^{-2}\end{array}$ & $\begin{array}{l}4.2 \times 10^{-2} \\
3.8 \times 10^{-2}\end{array}$ \\
\hline (1) & $1.41 \times 10^{-8}$ & $-10^{2}$ & $\begin{array}{l}\text { THD } \\
\text { LFL }\end{array}$ & $\begin{array}{l}7.5 \times 10^{-2} \\
6.0 \times 10^{-2}\end{array}$ & $\begin{array}{l}1.1 \times 10^{-3} \\
7.9 \times 10^{-4}\end{array}$ & $\begin{array}{l}3.6 \times 10^{-2} \\
3.1 \times 10^{-2}\end{array}$ & $\begin{array}{l}4.5 \times 10^{-2} \\
3.8 \times 10^{-2}\end{array}$ \\
\hline (2) & $1.70 \times 10^{-8}$ & $-10^{3}$ & $\begin{array}{l}\text { THD } \\
\text { LFL }\end{array}$ & $\begin{array}{l}7.6 \times 10^{-2} \\
6.5 \times 10^{-4}\end{array}$ & $\begin{array}{l}1.1 \times 10^{-3} \\
8.6 \times 10^{-4}\end{array}$ & $\begin{array}{l}3.6 \times 10^{-2} \\
3.2 \times 10^{-2}\end{array}$ & $\begin{array}{l}4.6 \times 10^{-2} \\
3.9 \times 10^{-2}\end{array}$ \\
\hline (3) & $3.98 \times 10^{-8}$ & $-10^{4}$ & $\begin{array}{l}\text { THD } \\
\text { LFL }\end{array}$ & $\begin{array}{l}1.2 \times 10^{-3} \\
2.8 \times 10^{-3}\end{array}$ & $\begin{array}{l}1.8 \times 10^{-3} \\
3.8 \times 10^{-3}\end{array}$ & $\begin{array}{l}5.1 \times 10^{-2} \\
9.0 \times 10^{-2}\end{array}$ & $\begin{array}{l}6.5 \times 10^{-2} \\
1.0 \times 10^{-1}\end{array}$ \\
\hline (4) & $10^{-4}$ & $-4.27 \times 10^{5}$ & $\begin{array}{l}\text { THD } \\
\text { LFL }\end{array}$ & $\begin{array}{l}3.6 \times 10^{-3} \\
4.1 \times 10^{3}\end{array}$ & $\begin{array}{l}9.3 \times 10^{-4} \\
5.1 \times 10^{3}\end{array}$ & $\begin{array}{l}3.5 \times 10^{-2} \\
1.1 \times 10^{5}\end{array}$ & $\begin{array}{l}4.4 \times 10^{-2} \\
1.1 \times 10^{5}\end{array}$ \\
\hline
\end{tabular}




$$
\begin{aligned}
\frac{\Delta n_{s}}{n_{s}} & :=\frac{\left|n_{s}-n_{s, \mathrm{ADM}}\right|}{n_{s, \mathrm{ADM}}}, \\
\frac{\Delta r}{r} & :=\frac{\left|r-r_{\mathrm{ADM}}\right|}{r_{\mathrm{ADM}}}
\end{aligned}
$$

where $n_{s, \mathrm{ADM}}$ and $r_{\mathrm{ADM}}$ are the values obtained in the full theory by use of the ADM formalism.

Our model is included in the class of generalized Higgs inflation models[19]. In their model, they provide one simplified formula under some approximation. Using it, we also calculate $n_{s}$ and $r$ in our model, which are given in Appendix B 3. The accuracies of the perturbations are summarized in Table I. As the coupling parameters approach the region near the "conventional" Higgs inflation, the deviations from the "correct" values increase. The approximation used in [19] is broken near the "conventional" Higgs inflation model. On the other hand, our approximation method is still valid even in such parameter regions.

\section{SUMMARY AND DISCUSSION}

In this paper we propose a hybrid type of the conventional Higgs inflation and new Higgs inflation models. Performing a disformal transformation into the Einstein frame and truncating the higher-derivative terms of the Higgs field, we analyze the inflationary background dynamics and the cosmological perturbations. From the observed power spectrum of the density perturbations, we show the constraint on the nonminimal coupling constant $\xi$ and the mass parameter $M$ in the derivative coupling. Although the primordial tilt $n_{s}$ in the hybrid model barely changes, the tensor-to-scalar ratio $r$ moves from the value in new Higgs inflationary model to that in the conventional Higgs inflationary model as $|\xi|$ increases. $r$ varies in the range of the observationally arrowed region. Hence once we know the tensor-scalar ratio $r$ by the future observations, we can fix the coupling parameters $M$ and $\xi$. We confirm our results by numerical analysis by ADM formalism of the full theory in the Jordan frame. The higherderivative terms of the Higgs field appeared in the Einstein fame can become negligible because of the slow-rolling evolution during inflation.

As we mentioned in Introduction, our studied model is no more than the tree level. At the high-energy scale, such as an inflationary stage, we should take into account the quantum loop effects to obtain the effective potential. Since the loop corrections significantly change the effective Higgs potential via the running of the coupling constants, the conventional Higgs inflation model has been analyzed including in the loop corrections $[14,29,58]$. The frame dependence of the effective potential has also been discussed $[15,58]$. This problem is caused by which frame (either in the Jordan frame or in the Einstein frame) we use to quantize the Higgs field and calculate the effective potential. They found that $r$ would be enhanced so much that some parameter regions can be excluded by the observations. However, as we expect, the result should not depend on the frame choice $[41,59]$. Since we also have the stability problem of the Higgs field, we have to consider the loop corrections more carefully. We expect not only the Higgs coupling $\lambda$ but also the nonminimal coupling $\xi$ as well as the derivative coupling $M$ are running via renormalization. Such a calculation could be performed by the asymptotic safety approach [60-63]. These problems are under investigation.

\section{ACKNOWLEDGMENTS}

K. M. would like to thank Department of Physics, University of Auckland, for its hospitality during his visit in 2016, where the preliminary work was started. He also acknowledges Richard Easther and Nathan Musoke for the useful discussions. S. S. would like to thank Hiroyuki Abe, Katsuki Aoki, Mitsuhiro Fukushima, Yuta Hamada, Kohei Kamada, and Yosuke Misonoh for valuable comments and fruitful discussions. This work was supported in part by JSPS KAKENHI Grants No. JP16K05362 and No. JP17H06359.

\section{APPENDIX A: TOWARDS THE EINSTEIN FRAME VIA DISFORMAL TRANSFORMATION}

\section{Disformal transformation}

We first consider the following disformal transformation,

$$
g_{\mu \nu}=\bar{\Omega}^{2}\left(\bar{g}_{\mu \nu}+\bar{u}_{\mu} \bar{u}_{\nu},\right)
$$

from the original metric $\bar{g}_{\mu \nu}$ with a vector field $\bar{u}^{\mu}$, which is either timelike or spacelike, i.e.,

$$
\bar{u}^{\mu} \bar{u}_{\mu}=\epsilon \bar{u}^{2},
$$

where $\epsilon= \pm 1$. This transformation leads to

$$
\begin{aligned}
\sqrt{-g} & =\bar{\Omega}^{D}\left(1+\epsilon \bar{u}^{2}\right)^{\frac{1}{2}} \sqrt{-\bar{g}} \\
g^{\mu \nu} & =\bar{\Omega}^{-2}\left(\bar{g}^{\mu \nu}-\frac{1}{1+\epsilon \bar{u}^{2}} \bar{u}^{\mu} \bar{u}^{\nu}\right) \\
\Gamma_{\nu \rho}^{\mu} & =\bar{\Gamma}_{\nu \rho}^{\mu}+\bar{\gamma}_{\nu \rho}^{\mu}
\end{aligned}
$$

where $D$ is the spacetime dimension, and the deviation of the connection $\bar{\gamma}_{\nu \rho}^{\mu}$ is given by

$$
\bar{\gamma}_{\nu \rho}^{\mu}=\bar{f}_{\nu \rho}^{\mu}+\bar{\omega}_{\nu \rho}^{\mu}
$$

with 


$$
\begin{aligned}
\bar{f}_{\rho \sigma}^{\mu}:= & \frac{1}{2}\left(\bar{g}^{\mu \nu}-\frac{1}{1+\epsilon \bar{u}^{2}} \bar{u}^{\mu} \bar{u}^{\nu}\right) \\
& \times\left[\bar{\nabla}_{\rho}\left(\bar{u}_{\nu} \bar{u}_{\sigma}\right)+\bar{\nabla}_{\sigma}\left(\bar{u}_{\nu} \bar{u}_{\rho}\right)-\bar{\nabla}_{\nu}\left(\bar{u}_{\rho} \bar{u}_{\sigma}\right)\right] \\
\bar{\omega}_{\rho \sigma}^{\mu}:= & \delta_{\rho}^{\mu} \bar{\nabla}_{\sigma} \ln \bar{\Omega}+\delta_{\sigma}^{\mu} \bar{\nabla}_{\rho} \ln \bar{\Omega}-\left(\bar{g}^{\mu \nu}-\frac{1}{1+\epsilon \bar{u}^{2}} \bar{u}^{\mu} \bar{u}^{\nu}\right) \\
& \times\left(\bar{g}_{\rho \sigma}+\bar{u}_{\rho} \bar{u}_{\sigma}\right) \bar{\nabla}_{\nu} \ln \bar{\Omega},
\end{aligned}
$$

where $\bar{\nabla}^{\mu}$ denotes the covariant derivative with respect to the metric $\bar{g}_{\mu \nu}$.

The Riemann, Ricci, and scalar curvatures are given by

$$
\begin{aligned}
R_{\nu \rho \sigma}^{\mu}= & \bar{R}_{\nu \rho \sigma}^{\mu}+\bar{\nabla}_{\rho} \bar{\gamma}_{\nu \sigma}^{\mu}-\bar{\nabla}_{\sigma} \bar{\gamma}_{\nu \rho}^{\mu}+\bar{\gamma}_{\alpha \sigma}^{\mu} \bar{\gamma}_{\nu \rho}^{\alpha}-\bar{\gamma}_{\alpha \rho}^{\mu} \bar{\gamma}_{\nu \sigma}^{\alpha} \\
R_{\rho \sigma}= & \bar{R}_{\rho \sigma}+\bar{\nabla}_{\mu} \bar{\gamma}_{\rho \sigma}^{\mu}-\bar{\nabla}_{\sigma} \bar{\gamma}_{\mu \rho}^{\mu}+\bar{\gamma}_{\mu \alpha}^{\mu} \bar{\gamma}_{\rho \sigma}^{\alpha}-\bar{\gamma}_{\alpha \rho}^{\beta} \bar{\gamma}_{\beta \sigma}^{\alpha} \\
R= & \bar{\Omega}^{-2}\left[\frac{2+\epsilon \bar{u}^{2}}{2\left(1+\epsilon \bar{u}^{2}\right)} \bar{R}-\frac{1}{1+\epsilon \bar{u}^{2}} \bar{G}_{\mu \nu} \bar{u}^{\mu} \bar{u}^{\nu}+\bar{\nabla}_{\mu}\left(\bar{g}^{\rho \sigma} \bar{\gamma}_{\rho \sigma}^{\mu}\right)\right. \\
& -\bar{\nabla}^{\rho} \bar{\gamma}_{\mu \rho}^{\mu}+\bar{g}^{\rho \sigma} \bar{\gamma}_{\rho \sigma}^{\alpha} \bar{\gamma}_{\mu \alpha}^{\mu}-\bar{g}^{\rho \sigma} \bar{\gamma}_{\alpha \rho}^{\beta} \bar{\gamma}_{\beta \sigma}^{\alpha}-\frac{1}{1+\epsilon \bar{u}^{2}} \bar{u}^{\rho} \bar{u}^{\sigma} \\
& \left.\times\left(\bar{\nabla}_{\mu} \bar{\gamma}_{\rho \sigma}^{\mu}-\bar{\nabla}_{\sigma} \bar{\gamma}_{\mu \rho}^{\mu}+\bar{\gamma}_{\mu \alpha}^{\mu} \bar{\gamma}_{\rho \sigma}^{\alpha}-\bar{\gamma}_{\alpha \rho}^{\beta} \bar{\gamma}_{\beta \sigma}^{\alpha}\right)\right] .
\end{aligned}
$$

Hence, we obtain the Einstein tensor as

$$
\begin{aligned}
G_{\mu \nu}= & \bar{G}_{\mu \nu}+\frac{1}{2\left(1+\epsilon \bar{u}^{2}\right)}\left(\bar{g}_{\mu \nu}+\bar{u}_{\mu} \bar{u}_{\nu}\right) \bar{u}^{\alpha} \bar{u}^{\beta} \bar{G}_{\alpha \beta} \\
& +\frac{\epsilon \bar{u}^{2}}{4\left(1+\epsilon \bar{u}^{2}\right)}\left(\bar{g}_{\mu \nu}+\bar{u}_{\mu} \bar{u}_{\nu}\right) \bar{R}-\frac{1}{2} \bar{u}_{\mu} \bar{u}_{\nu} \bar{R} \\
& +\bar{\nabla}_{\rho} \bar{\gamma}_{\mu \nu}^{\rho}-\bar{\nabla}_{\nu} \bar{\gamma}_{\rho \mu}^{\rho}+\bar{\gamma}_{\rho \sigma}^{\rho} \bar{\gamma}_{\mu \nu}^{\sigma}-\bar{\gamma}_{\rho \mu}^{\sigma} \bar{\gamma}_{\sigma \nu}^{\rho}-\frac{1}{2}\left(\bar{g}_{\mu \nu}+\bar{u}_{\mu} \bar{u}_{\nu}\right) \\
& \times\left[\bar{\nabla}_{\alpha}\left(\bar{g}^{\rho \sigma} \bar{\gamma}_{\rho \sigma}^{\alpha}\right)-\bar{\nabla}^{\rho} \bar{\gamma}_{\sigma \rho}^{\sigma}+\bar{g}^{\rho \sigma} \bar{\gamma}_{\rho \sigma}^{\alpha} \bar{\gamma}_{\beta \alpha}^{\beta}-\bar{g}^{\rho \sigma} \bar{\gamma}_{\alpha \rho}^{\beta} \bar{\gamma}_{\beta \sigma}^{\alpha}\right. \\
& \left.-\frac{1}{1+\epsilon \bar{u}^{2}} \bar{u}^{\rho} \bar{u}^{\sigma}\left(\bar{\nabla}_{\alpha} \bar{\gamma}_{\rho \sigma}^{\alpha}-\bar{\nabla}_{\sigma} \bar{\gamma}_{\alpha \rho}^{\alpha}+\bar{\gamma}_{\beta \alpha}^{\beta} \bar{\gamma}_{\rho \sigma}^{\alpha}-\bar{\gamma}_{\alpha \rho}^{\beta} \bar{\gamma}_{\beta \sigma}^{\alpha}\right)\right] .
\end{aligned}
$$

\section{Towards the Einstein frame}

Now we consider the following action,

$S=\int d^{D} x \sqrt{-g}\left[F_{4}(\phi) R(\tilde{g})+F_{5}(\phi) G^{\mu \nu}(g) \nabla_{\mu} \phi \nabla_{\nu} \phi\right]$,

where $F_{4}(\phi)$ and $F_{5}(\phi)$ are arbitrary functions of a scalar field $\phi$. This gives a gravity sector of some simple scalartensor gravity theory.

Performing the disformal transformation (A1) with $\bar{u}_{\mu}=\beta \bar{\nabla}_{\mu} \phi$, we find

$$
R=\bar{\Omega}^{-2}\left[\frac{2+\epsilon \bar{u}^{2}}{2\left(1+\epsilon \bar{u}^{2}\right)} \bar{R}-\frac{1}{1+\epsilon \bar{u}^{2}} \bar{G}_{\mu \nu} \bar{u}^{\mu} \bar{u}^{\nu}+\cdots\right]
$$

$$
\begin{aligned}
G_{\mu \nu}= & \bar{G}_{\mu \nu}+\frac{1}{2\left(1+\epsilon \bar{u}^{2}\right)}\left(\bar{g}_{\mu \nu}+\bar{u}_{\mu} \bar{u}_{\nu}\right) \bar{u}^{\alpha} \bar{u}^{\beta} \bar{G}_{\alpha \beta} \\
& -\frac{\bar{u}^{2}}{4\left(1+\epsilon \bar{u}^{2}\right)}\left(\bar{g}_{\mu \nu}+\bar{u}_{\mu} \bar{u}_{\nu}\right) \bar{R}-\frac{1}{2} \bar{u}_{\mu} \bar{u}_{\nu} \bar{R}+\cdots,
\end{aligned}
$$

where $\cdots$ describes some functions of $\phi$ and its derivatives. Since $\nabla_{\mu} \phi=\partial_{\mu} \phi=\bar{\nabla}_{\mu} \phi$, we find the action (A3) as

$$
\begin{aligned}
S= & \int d^{D} x \sqrt{-\bar{g}}[A(\bar{\Omega}, \beta) \bar{R}(\bar{g})+B(\bar{\Omega}, \beta) \\
& \left.\times \bar{G}_{\alpha \beta}(\bar{g}) \bar{\nabla}^{\alpha} \phi \bar{\nabla}^{\beta} \phi+\cdots\right],
\end{aligned}
$$

where

$$
\begin{aligned}
A= & \frac{\bar{\Omega}^{D-4}\left(1+\epsilon \bar{u}^{2}\right)^{-3 / 2}}{4 \beta^{2}}\left[2 \beta^{2} \bar{\Omega}^{2}\left(1+\epsilon \bar{u}^{2}\right)\left(2+\epsilon \bar{u}^{2}\right)\right. \\
& \left.\times F_{4}-\left(\epsilon \bar{u}^{2}\right)^{2} F_{5}\right] \\
B= & \frac{\bar{\Omega}^{D-4}\left(1+\epsilon \bar{u}^{2}\right)^{-3 / 2}}{2}\left[-2 \beta^{2} \bar{\Omega}^{2}\left(1+\epsilon \bar{u}^{2}\right) F_{4}\right. \\
& \left.+\left(2+\epsilon \bar{u}^{2}\right) F_{5}\right] .
\end{aligned}
$$

In order to find only the Einstein-Hilbert action in the $\bar{g}$ frame, we have to impose $A=\frac{1}{2}$ and $B=0$. We then find the equations for $\bar{\Omega}$ and $\beta$ as

$2 \beta^{2} \bar{\Omega}^{2}\left(1+\epsilon \bar{u}^{2}\right)\left(2+\epsilon \bar{u}^{2}\right) F_{4}-\left(\epsilon \bar{u}^{2}\right)^{2} F_{5}=2 \beta^{2} \bar{\Omega}^{4-D}\left(1+\epsilon \bar{u}^{2}\right)^{3 / 2}$

$-2 \beta^{2} \bar{\Omega}^{2}\left(1+\epsilon \bar{u}^{2}\right) F_{4}+\left(2+\epsilon \bar{u}^{2}\right) F_{5}=0$.

These equations fix $\bar{\Omega}$ and $\beta$ as

$$
\bar{\Omega}^{D-2}=\frac{\left(2+\epsilon \bar{u}^{2}\right)}{4\left(1+\epsilon \bar{u}^{2}\right)^{1 / 2} F_{4}}
$$

$$
\beta^{2}=\frac{\left(2+\epsilon \bar{u}^{2}\right) F_{5}}{2\left(1+\epsilon \bar{u}^{2}\right) F_{4}} \bar{\Omega}^{-2} .
$$

Since $\epsilon \bar{u}^{2}=\beta^{2}(\nabla \phi)^{2}$, we have to solve the coupled equations (A4) and (A5) to describe $\bar{\Omega}$ and $\beta$ explicitly in terms of $\phi$ and $\nabla \phi$.

As a result, the disformal transformation (A1) with (A4) and (A5) gives the Einstein gravity in the $\bar{g}$ frame, which we call the Einstein frame.

\section{Hybrid Higgs inflation}

Next we apply the above result to the hybrid Higgs inflation model in four dimensions $(D=4)$, which is given by 


$$
\begin{aligned}
S= & \int d^{4} x \sqrt{-g}\left[\frac{1-\xi \phi^{2}}{2} R\right. \\
& \left.-\frac{1}{2}\left(g^{\mu \nu}-\frac{G^{\mu \nu}}{M^{2}}\right) \nabla_{\mu} \phi \nabla_{\nu} \phi-V(\phi)\right] .
\end{aligned}
$$

Since we are interested in a cosmological dynamics, for the disformal transformation,

$$
g_{\mu \nu}=\bar{\Omega}^{2}\left(\bar{g}_{\mu \nu}+\beta^{2} \bar{\nabla}_{\mu} \phi \bar{\nabla}_{\nu} \phi\right),
$$

we choose a timelike vector $\bar{u}_{\alpha}=\beta \bar{\nabla}_{\mu} \phi$ with $\bar{u}^{\alpha} \bar{u}_{\alpha}=$ $-\bar{u}^{2}(\epsilon=-1)$.

To find the Einstein gravity in the transformed $\bar{g}$ frame, we choose

$$
\begin{aligned}
\bar{\Omega}^{2} & =\frac{\left(2-\bar{u}^{2}\right)}{2\left(1-\bar{u}^{2}\right)^{\frac{1}{2}}\left(1-\xi \phi^{2}\right)} \\
\beta^{2} & =\frac{1}{\left(1-\bar{u}^{2}\right)^{\frac{1}{2}} M^{2}} .
\end{aligned}
$$

Introducing the canonical kinetic term of the Higgs field,

$$
\bar{X}:=-\frac{1}{2} \bar{g}^{\mu \nu} \bar{\nabla}_{\mu} \phi \bar{\nabla}_{\nu} \phi,
$$

since $\bar{u}^{2}=-\bar{u}^{\alpha} \bar{u}_{\alpha}=2 \beta^{2} \bar{X}$, we find the relation between $\bar{u}$ and $\bar{X}$ as

$$
\bar{u}^{2}\left(1-\bar{u}^{2}\right)^{\frac{1}{2}}=\frac{2 \bar{X}}{M^{2}} .
$$

The gravity action is now given by the Einstein-Hilbert term. How about the action of the Higgs field? Apart from the standard action in the original frame, we have the additional contribution $L_{\text {disformal }}$ from the disformal transformation as

$S_{\text {Higgs }}=\int d^{4} x \sqrt{-g}\left[-\frac{1}{2} g^{\mu \nu} \nabla_{\mu} \phi \nabla_{\nu} \phi-V(\phi)+L_{\text {disformal }}\right]$,

where

$$
\begin{aligned}
L_{\text {disformal }}= & \frac{1-\xi \phi^{2}}{2 \bar{\Omega}^{2}}\left[\bar{\nabla}_{\mu}\left(\bar{g}^{\rho \sigma} \bar{\gamma}_{\rho \sigma}^{\mu}\right)-\bar{\nabla}^{\rho} \bar{\gamma}_{\mu \rho}^{\mu}+\bar{g}^{\rho \sigma} \bar{\gamma}_{\rho \sigma}^{\alpha} \bar{\gamma}_{\mu \alpha}^{\mu}-\bar{g}^{\rho \sigma} \bar{\gamma}_{\alpha \rho}^{\beta} \bar{\gamma}_{\beta \sigma}^{\alpha}\right. \\
& \left.-\frac{\beta^{2}}{1-\bar{u}^{2}} \bar{\nabla}^{\rho} \phi \bar{\nabla}^{\sigma} \phi\left(\bar{\nabla}_{\mu} \bar{\gamma}_{\rho \sigma}^{\mu}-\bar{\nabla}_{\sigma} \bar{\gamma}_{\mu \rho}^{\mu}+\bar{\gamma}_{\mu \alpha}^{\mu} \bar{\gamma}_{\rho \sigma}^{\alpha}-\bar{\gamma}_{\alpha \rho}^{\beta} \bar{\gamma}_{\beta \sigma}^{\alpha}\right)\right] \\
& +\frac{\bar{g}^{\mu \lambda} \bar{g}^{\nu \kappa} \bar{\nabla}_{\lambda} \phi \bar{\nabla}_{k} \phi}{2 M^{2}}\left[\bar{\nabla}_{\rho} \bar{\gamma}_{\mu \nu}^{\rho}-\bar{\nabla}_{\nu} \bar{\gamma}_{\rho \mu}^{\rho}+\bar{\gamma}_{\rho \sigma}^{\rho} \bar{\gamma}_{\mu \nu}^{\sigma}-\bar{\gamma}_{\rho \mu}^{\sigma} \bar{\gamma}_{\sigma \nu}^{\rho}\right. \\
& -\frac{1}{2}\left(\bar{g}_{\mu \nu}+\beta^{2} \bar{\nabla}_{\mu} \phi \bar{\nabla}_{\nu} \phi\right)\left[\bar{\nabla}_{\alpha}\left(\bar{g}^{\rho \sigma} \bar{\gamma}_{\rho \sigma}^{\alpha}\right)-\bar{\nabla}^{\rho} \bar{\gamma}_{\sigma \rho}^{\sigma}+\bar{g}^{\rho \sigma} \bar{\gamma}_{\rho \sigma}^{\alpha} \bar{\gamma}_{\beta \alpha}^{\beta}-\bar{g}^{\rho \sigma} \bar{\gamma}_{\alpha \rho}^{\beta} \bar{\gamma}_{\beta \sigma}^{\alpha}\right. \\
& \left.-\frac{\beta^{2}}{1-\bar{u}^{2}} \bar{\nabla}^{\rho} \phi \bar{\nabla}^{\sigma} \phi\left(\bar{\nabla}_{\alpha} \bar{\gamma}_{\rho \sigma}^{\alpha}-\bar{\nabla}_{\sigma} \bar{\gamma}_{\alpha \rho}^{\alpha}+\bar{\gamma}_{\beta \alpha}^{\beta} \bar{\gamma}_{\rho \sigma}^{\alpha}-\bar{\gamma}_{\alpha \rho}^{\beta} \bar{\gamma}_{\beta \sigma}^{\alpha}\right)\right] .
\end{aligned}
$$

In order to obtain the action in the Einstein frame, we have to rewrite the variables in the $g$ frame to those in the $\bar{g}$ frame. Since $L_{\text {disformal }}$ is already given by the variables in the $\bar{g}$ frame, we easily find the action of the Higgs field in the Einstein frame as

$$
\begin{aligned}
S_{\mathrm{Higgs}}= & \int d^{4} x \sqrt{-\bar{g}} \bar{\Omega}^{4}\left(1-\bar{u}^{2}\right)^{\frac{1}{2}} \\
& \times\left[-\frac{1}{2} \bar{\Omega}^{-2} \bar{g}^{\mu \nu} \bar{\nabla}_{\mu} \phi \bar{\nabla}_{\nu} \phi-V(\phi)+L_{\text {disformal }}\right] .
\end{aligned}
$$

Since the above additional term $L_{\text {disformal }}$ is very complicated, it seems not to be useful for a practical purpose. However, since we are interested in an inflationary era, at which the scalar field changes very slowly, the kinetic term $\bar{X}$ is small in the slow-rolling phase and then we may be able to ignore the higher-derivative terms such as $\square \phi$ and $\bar{X}^{2}$. The resultant action becomes much simpler. We shall show below what we find as the lowest order.
Expanding Eq. (A6) in terms of $\bar{X}$, we find

$$
\bar{u}^{2}=\frac{2 \bar{X}}{M^{2}}\left(1+\frac{\bar{X}}{M^{2}}+\cdots\right) .
$$

The kinetic term and the potential term in the standard Higgs action are rewritten by the similar expansion as

$$
\begin{aligned}
-\frac{1}{2} \sqrt{-g}(\nabla \phi)^{2} & =-\frac{1}{2} \sqrt{-g}(\bar{\nabla} \phi)^{2} \times \frac{\left[1-\frac{(\bar{\nabla} \phi)^{2}}{2 M^{2}}+\cdots\right]}{1-\xi \phi^{2}} \\
-\sqrt{-g} V(\phi) & =-\sqrt{-\bar{g}} \frac{V(\phi)}{\left(1-\xi \phi^{2}\right)^{2}}\left[1+\frac{(\bar{\nabla} \phi)^{2}}{2 M^{2}}-\frac{(\bar{\nabla} \phi)^{4}}{8 M^{4}} \cdots\right] .
\end{aligned}
$$

The additional term $L_{\text {disformal }}$, in which both $\bar{f}_{\nu \rho}^{\mu}$ and $\bar{\omega}_{\nu \rho}^{\mu}$ are the functionals of $\phi$ and its derivatives, is very complicated. However, $\bar{f}_{\nu \rho}^{\mu}$ consists only of the derivatives 
of $\bar{u}^{\mu}=\beta \bar{\nabla}^{\mu} \phi$, which are the higher-derivative terms of $\phi$. We may neglect such terms. On the other hand, $\bar{\omega}_{\nu \rho}^{\mu}$ contains the derivative of the conformal factor $\bar{\Omega}$. Hence, its contribution provides the square of the first derivative term of the scalar field $\phi$, which gives the lowest order in the expansion. Hence, we have to pick up the relevant contributions from the terms with $\bar{\omega}_{\nu \rho}^{\mu}$. Since the derivatives of $\bar{\omega}_{\nu \rho}^{\mu}$ are the higher derivatives of $\phi$, only algebraic terms remain.

We then find

$$
\begin{aligned}
\sqrt{-g} L_{\text {disformal }}= & \sqrt{-\bar{g}} \bar{\Omega}^{4}\left(1-\bar{u}^{2}\right)^{\frac{1}{2}} \frac{1-\xi \phi^{2}}{2 \bar{\Omega}^{2}} \\
& \times\left[\bar{g}^{\rho \sigma} \bar{\omega}^{\alpha}{ }_{\rho \sigma} \bar{\omega}_{\mu \alpha}^{\mu}-\bar{g}^{\rho \sigma} \bar{\omega}^{\beta}{ }_{\alpha \rho} \bar{\omega}_{\beta \sigma}^{\alpha}+\cdots\right] \\
= & \sqrt{-\bar{g}}\left[-3 \frac{\xi^{2} \phi^{2}}{\left(1-\xi \phi^{2}\right)^{2}}(\bar{\nabla} \phi)^{2}+\cdots\right] .
\end{aligned}
$$

As a result, the effective lowest-order action for the Higgs field in the Einstein frame is given by

$$
\begin{aligned}
S_{\mathrm{Higgs}}= & -\int d^{4} x \sqrt{-\bar{g}}\left[\frac{1}{2}\left(\frac{1-\xi(1-6 \xi) \phi^{2}+\frac{V(\phi)}{M^{2}}}{\left(1-\xi \phi^{2}\right)^{2}}\right)(\bar{\nabla} \phi)^{2}\right. \\
& \left.+\frac{V(\phi)}{\left(1-\xi \phi^{2}\right)^{2}}+\cdots\right] \\
\approx & -\int d^{4} x \sqrt{-\bar{g}}\left[\frac{1}{2}(\bar{\nabla} \Phi)^{2}+U(\Phi)+\cdots\right],
\end{aligned}
$$

where

$$
\begin{aligned}
\frac{d \Phi}{d \phi} & =\frac{\sqrt{1-\xi(1-6 \xi) \phi^{2}+\frac{V(\phi)}{M^{2}}}}{1-\xi \phi^{2}} \\
U(\Phi) & =\frac{V(\phi)}{\left(1-\xi \phi^{2}\right)^{2}} .
\end{aligned}
$$

\section{APPENDIX B: ANALYSIS IN JORDAN FRAME}

\section{The basic equations for a Friedmann universe in the Jordan frame}

We shall derive the basic equations for a background universe in the Jordan frame. We adopt the flat FLRW metric, as

$$
d s^{2}=-\mathcal{N}^{2} d t^{2}+a(t)^{2} d x^{2},
$$

where $\mathcal{N}$ is a lapse function and $a$ is a scale factor of the Universe. The action (2.1) is written as

$$
\begin{aligned}
S= & \int d^{4} x \mathcal{N} a^{3}\left[-3 \frac{H^{2}}{\mathcal{N}^{2}}\left(1-\xi \phi^{2}\right)+\left(1+\frac{3 H^{2}}{\mathcal{N}^{2} M^{2}}\right) \frac{\dot{\phi}^{2}}{2 \mathcal{N}^{2}}\right. \\
& \left.+6 \xi \frac{H \phi \dot{\phi}}{\mathcal{N}^{2}}-V(\phi)\right],
\end{aligned}
$$

where $H:=\dot{a} / a$ is the Hubble expansion parameter and a dot denotes the derivative with respect to the cosmic time $t$.

Taking the variation with respect to the lapse function $\mathcal{N}$, we find

$H^{2}=\frac{1}{3\left(1-\xi \phi^{2}\right)}\left[\left(1+\frac{9 H^{2}}{M^{2}}\right) \frac{\dot{\phi}^{2}}{2}+6 \xi \phi \dot{\phi} H+V(\phi)\right]$,

where we have set $\mathcal{N}=1$. This is just the Friedmann equation in the Jordan frame. The variation with respect to the scale factor $a$ gives

$$
\begin{gathered}
\left(3 H^{2}+2 \dot{H}\right)\left(1-\xi \phi^{2}\right)+\left(1-\frac{3 H^{2}}{M^{2}}-4 \xi\right) \frac{\dot{\phi}^{2}}{2}-V(\phi) \\
-\frac{1}{M^{2}} \frac{d}{d t}\left(H \dot{\phi}^{2}\right)-2 \xi \phi \ddot{\phi}-4 \xi \phi \dot{\phi} H=0 .
\end{gathered}
$$

The equation of motion of the Higgs field is given by variation with respect to $\phi$ as

$$
\begin{aligned}
& \left(1+\frac{3 H^{3}}{M^{2}}\right) \ddot{\phi}+3 H\left(1+\frac{3 H^{2}+2 \dot{H}}{M^{2}}\right) \dot{\phi}+6 \xi\left(\dot{H}+2 H^{2}\right) \phi \\
& +\frac{d V}{d \phi}=0 .
\end{aligned}
$$

Equations (B1), (B2), and (B3) are the basic equations for a Friedmann universe in the Jordan frame.

\section{Cosmological perturbations in the Jordan frame by use of the ADM formalism}

In order to check the accuracy of the perturbations calculated in the truncated model in the Einstein frame, we have to analyze the perturbations in the full theory. Although the disformal transformation provides the equivalent cosmological perturbations [51-56], the full action in the Einstein frame is too complicated to be analyzed. Hence, we perform the perturbations in the original Jordan frame by use of the ADM formalism [64]. We expect that this approach gives the correct perturbations. We then compare those with our results evaluated by the truncated model in the Einstein frame.

We use the unitary gauge, in which the Higgs field is uniform $\phi=\phi(t)$. The perturbed metric is written as

$$
d s^{2}=-\mathcal{N}^{2} d t^{2}+\gamma_{i j}\left(d x^{i}+\mathcal{N}^{i} d t\right)\left(d x^{j}+\mathcal{N}^{j} d t\right),
$$

where

$$
\begin{aligned}
\mathcal{N} & =1+\alpha, \quad \mathcal{N}_{i}=\partial_{i} \beta \\
\gamma_{i j} & =a^{2}(t) e^{2 \zeta}\left(\delta_{i j}+h_{i j}+\frac{1}{2} h_{i k} h_{k j}\right) .
\end{aligned}
$$

$\alpha, \beta, \zeta$, and $h_{i j}$ are the scalar modes and the tensor mode of perturbations, respectively. $h_{i j}$ satisfies the transversetraceless conditions $h_{i i}=h_{i j, j}=0$, 
First we consider the scalar perturbations. Varying the action with respect to the lapse $\alpha$ and the shift $\beta$, we find two constraint equations,

$$
\begin{aligned}
\alpha= & \frac{1-\xi \phi^{2}-\frac{\dot{\phi}^{2}}{2 M^{2}}}{H\left(1-\xi \phi^{2}-\frac{3 \dot{\phi}^{2}}{2 M^{2}}\right)-\xi \phi \dot{\phi}} \dot{\zeta}, \\
\frac{\partial_{i}^{2} \beta}{a^{2}}= & -\frac{1-\xi \phi^{2}-\frac{\dot{\phi}^{2}}{2 M^{2}}}{H\left(1-\xi \phi^{2}-\frac{3 \dot{\phi}^{2}}{2 M^{2}}\right)-\xi \phi \dot{\phi}} \frac{\partial_{i}^{2} \zeta}{a^{2}} \\
& +\left\{3+\frac{1-\xi \phi^{2}-\frac{\dot{\phi}^{2}}{2 M^{2}}}{\left[H\left(1-\xi \phi^{2}-\frac{3 \dot{\phi}^{2}}{2 M^{2}}\right)-\xi \phi \dot{\phi}\right]^{2}}\right. \\
& \left.\times\left[\frac{\dot{\phi}^{2}}{2}+6 H \xi \phi \dot{\phi}-3 H^{2}\left(1-\xi \phi^{2}-\frac{3 \dot{\phi}^{2}}{M^{2}}\right)\right]\right\} \dot{\zeta}
\end{aligned}
$$

Replacing $\alpha$ and $\beta$ with $\zeta$ by these constraints, we obtain the quadratic action for the scalar perturbations as

$$
S_{S}^{(2)}=\int d t d^{3} x a^{3}\left[G_{S} \dot{\zeta}^{2}-\frac{F_{S}}{a^{2}}\left(\partial_{i} \zeta\right)^{2}\right],
$$

where

$$
\begin{aligned}
G_{S}= & 3\left(1-\xi \phi^{2}-\frac{\dot{\phi}^{2}}{2 M^{2}}\right)+\frac{\left(1-\xi \phi^{2}-\frac{\dot{\phi}^{2}}{2 M^{2}}\right)^{2}}{\left[H\left(1-\xi \phi^{2}-\frac{3 \dot{\phi}^{2}}{2 M^{2}}\right)-\xi \phi \dot{\phi}\right]^{2}} \\
& \times\left[-3 H^{2}\left(1-\xi \phi^{2}-\frac{3 \dot{\phi}^{2}}{M^{2}}\right)+\frac{\dot{\phi}^{2}}{2}+6 H \xi \phi \dot{\phi}\right] \\
F_{S}= & \frac{1}{a} \frac{d}{d t}\left[\frac{a\left(1-\xi \phi^{2}-\frac{\dot{\phi}^{2}}{2 M^{2}}\right)^{2}}{H\left(1-\xi \phi^{2}-\frac{3 \dot{\phi}^{2}}{2 M^{2}}\right)-\xi \phi \dot{\phi}}\right] \\
& -\left(1-\xi \phi^{2}+\frac{\dot{\phi}^{2}}{2 M^{2}}\right) .
\end{aligned}
$$

The sound speed $c_{S}$ is given by

$$
c_{S}^{2}=\frac{F_{S}}{G_{S}} .
$$

We assume the following conditions during inflation:

$$
\begin{aligned}
\epsilon & :=-\frac{\dot{H}}{H^{2}} \simeq \text { const } \\
f_{S}: & : \frac{\dot{F}_{S}}{H F_{S}} \simeq \text { const } \\
g_{S} & :=\frac{\dot{G}_{S}}{H G_{S}} \simeq \text { const },
\end{aligned}
$$

which are confirmed by numerical analysis. The power spectrum of the scalar perturbations is given by

$$
\mathcal{P}_{\zeta}=\left.\frac{\gamma_{S}}{2} \sqrt{\frac{G_{S}}{F_{S}^{3}}} \frac{H^{2}}{4 \pi^{2}}\right|_{\text {horizon }},
$$

where

$$
\gamma_{S}=2^{\frac{2\left(3-\epsilon-g_{S}\right)}{2-2 \epsilon-f_{S} / 2+g_{S} / 2}-3}\left|\frac{\Gamma\left(\frac{3-\epsilon+g_{s}}{2-2 \epsilon-f_{S} / 2+g_{S} / 2}\right)}{\Gamma\left(\frac{3}{2}\right)}\right|^{2}\left(1-\epsilon-\frac{f_{S}+g_{S}}{2}\right)^{2} .
$$

$\Gamma$ is the Gamma function. The subscript "horizon" means that the perturbations are evaluated at the crossing of the sound horizon. The spectrum index is obtained by

$$
n_{s}-1=3-\frac{2\left(3-\epsilon+g_{s}\right)}{2-2 \epsilon-f_{S} / 2+g_{S} / 2} .
$$

The tensor perturbations are also calculated in the same manner as the scalar perturbations. The quadratic action for the tensor perturbations is

$$
S_{T}^{(2)}=\frac{1}{8} \int d t d^{3} \boldsymbol{x} a^{3}\left[G_{T}\left(\dot{h}_{i j}\right)^{2}-\frac{F_{T}}{a^{2}}\left(\partial_{k} h_{i j}\right)^{2}\right],
$$

where

$$
\begin{aligned}
G_{T} & =1-\xi \phi^{2}-\frac{\dot{\phi}^{2}}{2 M^{2}} \\
F_{T} & =1-\xi \phi^{2}+\frac{\dot{\phi}^{2}}{2 M^{2}} .
\end{aligned}
$$

We also assume the following conditions:

$$
\begin{aligned}
& f_{T}=\frac{\dot{F}_{T}}{H F_{T}} \simeq \text { const } \\
& g_{T}=\frac{\dot{G}_{T}}{H G_{T}} \simeq \text { const },
\end{aligned}
$$

which are also confirmed numerically.

The power spectrum for tensor perturbations is given by

$$
\mathcal{P}_{T}=\left.8 \gamma_{T} \sqrt{\frac{G_{T}}{F_{T}^{3}}} \frac{H^{2}}{4 \pi^{2}}\right|_{\text {horizon }},
$$

where

$\gamma_{T}:=2^{\frac{2\left(3-\epsilon+g_{T}\right)}{2-2 \epsilon-f_{T} / 2+g_{T} / 2}}-3\left|\frac{\Gamma\left(\frac{3-\epsilon+g_{T}}{2-2 \epsilon-f_{T} / 2+g_{T} / 2}\right)}{\Gamma\left(\frac{3}{2}\right)}\right|^{2}\left(1-\epsilon-\frac{f_{T}+g_{T}}{2}\right)^{2}$.

As a result, we obtain the tensor-to-scalar ratio $r$ by

$$
r=\frac{\mathcal{P}_{T}}{\mathcal{P}_{\zeta}} .
$$

We expect that those values give the correct ones, although we have to perform numerical calculation. We compare the results obtained by some approximations with 
the above "correct" values and then judge the validity of the approximations.

\section{Large friction limit}

Equations (B4) and (B5) are still complicated to evaluate. Hence, in [19], taking the large friction limit, they have given a simplified formula. Since our model is one of the generalized Higgs inflation models, we may apply their formula to our analysis.

Here we show the results for the hybrid Higgs inflation model by use of their formula. The power spectra of the scalar and tensor perturbations are given by

$$
\mathcal{P}_{\zeta} \simeq \frac{\lambda \phi^{6}}{768 \pi^{2}\left(1-\xi \phi^{2}\right)}\left(1+\frac{\lambda \phi^{4}}{4 M^{2}\left(1-\xi \phi^{2}\right)}\right),
$$

[1] A. A. Starobinsky, Phys. Lett. 91B, 99 (1980).

[2] A. H. Guth, Phys. Rev. D 23, 347 (1981).

[3] K. Sato, Mon. Not. R. Astron. Soc. 195, 467 (1981).

[4] S. W. Hawking, Phys. Lett. 115B, 295 (1982).

[5] A. H. Guth and S. Y. Pi, Phys. Rev. Lett. 49, 1110 (1982).

[6] A. A. Starobinsky, Phys. Lett. 117B, 175 (1982).

[7] V. F. Mukhanov and G. V. Chibisov, Zh. Eksp. Teor. Fiz. 83, 475 (1982) [Sov. Phys. JETP 56, 258 (1982)].

[8] J. Martin, C. Ringeval, and V. Vennin, Phys. Dark Universe 5-6, 75 (2014).

[9] B. Spokoiny, Phys. Lett. B 147, 39 (1984).

[10] K. Maeda, J. A. Stein-Schabes, and T. Futamase, Phys. Rev. D 39, 2848 (1989).

[11] T. Futamase and K. Maeda, Phys. Rev. D 39, 399 (1989).

[12] F. L. Bezrukov and M. Shaposhnikov, Phys. Lett. B 659, 703 (2008).

[13] F. L. Bezrukov, A. Magnin, and M. Shaposhnikov, Phys. Lett. B 675, 88 (2009).

[14] A. De Simone, M. P. Hertzberg, and F. Wilczek, Phys. Lett. B 678, 1 (2009).

[15] F. Bezrukov and M. Shaposhnikov, J. High Energy Phys. 07 (2009) 089.

[16] F. Bezrukov, Classical Quantum Gravity 30, 214001 (2013).

[17] C. Germani and A. Kehagias, Phys. Rev. Lett. 105, 011302 (2010).

[18] C. Germani and A. Kehagias, J. Cosmol. Astropart. Phys. 05 (2010) 019; 06 (2010) 01(E).

[19] K. Kamada, T. Kobayashi, T. Takahashi, M. Yamaguchi, and J. Yokoyama, Phys. Rev. D 86, 023504 (2012).

[20] G. Degrassi, S. Di Vita, J. Elias-Miro, J. R. Espinosa, G. F. Giudice, G. Isidori, and A. Strumia, J. High Energy Phys. 08 (2012) 098.

[21] P. B. Arnold, Phys. Rev. D 40, 613 (1989).

[22] G. W. Anderson, Phys. Lett. B 243, 265 (1990).

[23] P. B. Arnold and S. Vokos, Phys. Rev. D 44, 3620 (1991). while the primordial tilt $n_{s}$ and the tensor-to-scalar ratio $r$ are

$$
\begin{aligned}
n_{s}-1 \simeq & -\frac{8\left(3-2 \xi \phi^{2}\right)}{\phi^{2}\left(1-\xi \phi^{2}\right)}\left(1+\frac{\lambda \phi^{4}}{4 M^{2}\left(1-\xi \phi^{2}\right)}\right)^{-1} \\
& -2 \frac{\lambda \phi^{2}\left(2-\xi \phi^{2}\right)}{M^{2}\left(1-\xi \phi^{2}\right)^{2}}\left(1+\frac{\lambda \phi^{4}}{4 M^{2}\left(1-\xi \phi^{2}\right)}\right)^{-2} \\
r \simeq & \frac{128}{\phi^{2}\left(1-\xi \phi^{2}\right)}\left(1+\frac{\lambda \phi^{4}}{4 M^{2}\left(1-\xi \phi^{2}\right)}\right)^{-1} .
\end{aligned}
$$

The $e$-folding $N$ is given by solving Eqs. (B1), (B2), and (B3). Based on these formulas, we show the accuracies of the cosmological perturbations in Table I.
[24] J. R. Espinosa and M. Quiros, Phys. Lett. B 353, 257 (1995).

[25] D. Buttazzo, G. Degrassi, P. P. Giardino, G. F. Giudice, F. Sala, A. Salvio, and A. Strumia, J. High Energy Phys. 12 (2013) 089.

[26] J. Elias-Miro, J. R. Espinosa, G. F. Giudice, G. Isidori, A. Riotto, and A. Strumia, Phys. Lett. B 709, 222 (2012).

[27] M. Shaposhnikov, in Proceedings, 6th International Workshop on Top Quark Physics (TOP2013): Durbach, Germany, 2013 (2014), p. 233, http://inspirehep.net/record/ 1334580/files/materialDisplay.pdf.

[28] J. R. Espinosa, G. F. Giudice, E. Morgante, A. Riotto, L. Senatore, A. Strumia, and N. Tetradis, J. High Energy Phys. 09 (2015) 174.

[29] F. Bezrukov, J. Rubio, and M. Shaposhnikov, Phys. Rev. D 92, 083512 (2015).

[30] L. Delle Rose, C. Marzo, and A. Urbano, J. High Energy Phys. 05 (2016) 050.

[31] M. Sher, Phys. Rep. 179, 273 (1989).

[32] Y. Hamada, H. Kawai, K.-y. Oda, and S. C. Park, Phys. Rev. Lett. 112, 241301 (2014).

[33] A. O. Barvinsky, A. Yu. Kamenshchik, and I. P. Karmazin, Phys. Rev. D 48, 3677 (1993).

[34] A. O. Barvinsky, A. Yu. Kamenshchik, C. Kiefer, A. A. Starobinsky, and C. F. Steinwachs, Eur. Phys. J. C 72, 2219 (2012).

[35] Y. Abe, M. Horikoshi, and T. Inami, Communications in Physics 26, 229 (2017).

[36] L. Smolin, Phys. Lett. 93B, 95 (1980).

[37] C. P. Burgess, H. M. Lee, and M. Trott, J. High Energy Phys. 09 (2009) 103.

[38] J. L. F. Barbon and J. R. Espinosa, Phys. Rev. D 79, 081302 (2009).

[39] C. P. Burgess, H. M. Lee, and M. Trott, J. High Energy Phys. 07 (2010) 007.

[40] F. Bezrukov, A. Magnin, M. Shaposhnikov, and S. Sibiryakov, J. High Energy Phys. 01 (2011) 016. 
[41] D. P. George, S. Mooij, and M. Postma, J. Cosmol. Astropart. Phys. 02 (2014) 024.

[42] D. P. George, S. Mooij, and M. Postma, J. Cosmol. Astropart. Phys. 04 (2016) 006.

[43] J. Fumagalli and M. Postma, J. High Energy Phys. 05 (2016) 049.

[44] C. Germani, Y. Watanabe, and N. Wintergerst, J. Cosmol. Astropart. Phys. 12 (2014) 009.

[45] A. Escriva and C. Germani, Phys. Rev. D 95, 123526 (2017).

[46] J. Fumagalli, S. Mooij, and M. Postma, arXiv:1711.08761.

[47] K. Maeda, Phys. Rev. D 37, 858 (1988).

[48] K. Maeda, Phys. Rev. D 39, 3159 (1989).

[49] S. Sato, K. Maeda, R. Easther, and N. Musoke, Hybrid Higgs inflation, in Proceedings of 2016 Autumn Meeting of the Physical Society of Japan (2016).

[50] S. Di Vita and C. Germani, Phys. Rev. D 93, 045005 (2016).

[51] N. Makino and M. Sasaki, Prog. Theor. Phys. 86, 103 (1991).

[52] M. Minamitsuji, Phys. Lett. B 737, 139 (2014).

[53] S. Tsujikawa, J. Cosmol. Astropart. Phys. 15 (2015) 043.
[54] Y. Watanabe, A. Naruko, and M. Sasaki, Europhys. Lett. 111, 39002 (2015).

[55] G. Domènech, A. Naruko, and M. Sasaki, J. Cosmol. Astropart. Phys. 15 (2015) 067.

[56] H. Motohashi and J. White, J. Cosmol. Astropart. Phys. 16 (2016) 065.

[57] P. Ade, N. Aghanim, M. Arnaud, M. Ashdown, J. Aumont, C. Baccigalupi, A. Banday, R. Barreiro, J. Bartlett, N. Bartolo et al., Astron. Astrophys. 594, A13 (2016).

[58] Y. Hamada, H. Kawai, K.-y. Oda, and S. C. Park, Phys. Rev. D 91, 053008 (2015).

[59] Y. Hamada, H. Kawai, Y. Nakanishi, and K.-y. Oda, Phys. Rev. D 95, 103524 (2017).

[60] Z.-Z. Xianyu and H.-J. He, J. Cosmol. Astropart. Phys. 14 (2014) 083.

[61] K.-y. Oda and M. Yamada, Classical Quantum Gravity 33, 125011 (2016).

[62] Y. Hamada and M. Yamada, J. High Energy Phys. 08 (2017) 070.

[63] M. Atkins and X. Calmet, Phys. Lett. B 697, 37 (2011).

[64] T. Kobayashi, M. Yamaguchi, and J. Yokoyama, Prog. Theor. Phys. 126, 511 (2011). 